\title{
Expanded Decorrelating Detector with Reduced Noise Enhancement for Multipath Frequency-Selective Fading Channels
}

\author{
H. Zhou, P. Xiao, W. L. Woo, and B. S. Sharif \\ School of Electrical, Electronic and Computer Engineering, Merz Court, \\ Newcastle University, NE1 7RU \\ United Kingdom \\ Emails: \{hongwei.zhou, pei.xiao, w.l.woo, bayan.sharif\}@ncl.ac.uk
}

\begin{abstract}
A novel hybrid multiuser detection scheme that jointly uses linear and nonlinear interference suppression techniques is developed for high-speed direct-sequence code-division multiple-access (DS/CDMA) communications in multipath frequency-selective fading channels. The detector detects signals in a symbol-by-symbol style. Conventional decorrelating detectors suffer from the noise enhancement problem, and the problem becomes more serious for dispersive multipath channels. The proposed detector uses interference cancellation (IC) technology to reduce the rank of the expanded signal subspace and hence it enjoys the advantages of the expanded decorrelating detector in terms of complete multiple access interference (MAI) and intersymbol interference (ISI) suppression and avoids its disadvantage in terms of noise enhancement. Meanwhile, for this new detection strategy, a novel Kalman filter-based adaptive power estimation algorithm is developed. Computer simulation shows dramatic performance gain in the new detector compared with other existing methods.
\end{abstract}

Index Terms: DS-CDMA, multiuser detection, expanded decorrelating detector, interference cancellation, Kalman filter, amplitude estimation, multipath frequency-selective fading channel 


\section{Introduction}

Code-division multiple-access (CDMA) is the core technique in all 3G mobile communication standards. It is also used in satellite communications [1] and other wireless systems such as Wi-Fi [2] and ZigBee [3]. Undoubtedly, CDMA will continue to play a pivotal role in future generation of wireless communications. It is well known that CDMA communication systems are interference limited, that is, the system capacity is limited by the multiple access interference (MAI) [4]. In the recent decade, there have been significant efforts in developing multiuser detection technologies to overcome the MAI problem. The maximum likelihood sequence estimator is the optimum detector in terms of bit error rate (BER) performance [5], but its complexity grows exponentially with the number of users, which is prohibitive for practical implementation. Linear multiuser detectors, such as the decorrelating detector [4] [6] [7] and the minimum mean square error (MMSE) detector [8], are suboptimum solutions, but they have low complexity, which only grows linearly with the number of users. The adaptive detectors are preferable to their non-adaptive counterparts because they do not need online computation of the crosscorrelation matrix and can adapt to dynamic environment by learning from the received data. The adaptive MMSE detector introduced in [9] is derived using training sequence and the stochastic gradient descent training algorithm. This training-based method does not require users' spreading codes, and is therefore suitable for downlink implementation. The need for training sequence is further avoided in the blind multiuser detection scheme proposed in [10], where a blind MMSE detector is developed by minimizing the mean output energy (MOE) subject to an orthogonality constraint. Its adaptive implementation is realized by using the least-mean-square (LMS) method. Another approach to blind detection is based on subspace estimation. In [11], the decorrelating detector and the MMSE detector are expressed in closed forms of the signal subspace components, and the signal subspace is tracked by using the project approximation subspace tracking deflation (PASTd) algorithm. This subspace approach is extended to the antenna array system in [12], resulting in a blind subspace-based spatio-temporal decorrelator and spatio-temporal MMSE detector. In [13] and [14], the blind detectors are expressed as variables in the signal subspace and the coefficients are adaptively derived by LMS and Kalman filtering methods, respectively. Parallel to the development of the linear detectors, there are also numerous efforts in developing interference cancellation (IC) technologies [4] [15] [16]. IC is a category of nonlinear methods that subtract the estimated interfering signals when demodulating the signal of the interested user. There are basically two classes of IC techniques- 
successive interference cancellation (SIC) [15] which subtracts interference in a sequential order, one user at a time; and parallel interference cancellation (PIC) [16] which subtracts the interference from all interfering users simultaneously. IC detectors are bootstrapping technologies- correct decisions will lead to correct recreation and subtraction of the interfering signals at the receiver, while wrong decisions will double the contribution of the interferer, so conventional IC methods are only suitable for high SNR environments.

In practical high-speed wireless communications, the intersymbol interference (ISI) caused by multipath propagation imposes significant concern. One way to overcome the effect of ISI is to employ multicarrier technique to transform a frequency selective fading channel to flat fading channels [17] [18] [19]. However, since the multipath signals become non-resolvable, the multicarrier approaches forgo the opportunity to make use of the effective multipath diversity gain. RAKE receiver is commonly used to solve the multipath problem [20], but it is optimum only in single-user systems. For multiuser systems, the RAKE structure can still be used but the matched filters in the front end are replaced by linear multiuser detectors [21] [22] [23]. In [24], the MOE detector in [10] is extended into multipath channel by using a Min/Max approach to simultaneously optimize the output energy and a constraint vector. The optimal constraint vector is a biased estimate of the channel vector. The estimation error is proportional to the channel noise and it causes inferior performance compared with the MMSE detector. In [25], the affect of noise is mitigated by using a Power of R (POR) method to virtually increase the SNR. Emphasis of this paper is the decorrelating detectors, which have been proposed in [6] [7] and [11] for multiuser detection in AWGN channel. For multipath frequencyselective fading channel, the ISI is modelled as virtual MAI in [26] and hence an expanded decorrelating detector is derived to simultaneously suppress MAI and ISI. The expanded decorrelating detectors are not limited by the channel length. Meanwhile, they maintain the desirable properties of distributed implementation and having no detection delays. However, all decorrelating detectors have the disadvantage of noise enhancement, and the expanded decorrelating detector operating in a higherrank subspace amplifies the noise even more significantly. This paper aims at solving this problem by using a hybrid detection strategy that combines linear and nonlinear methods to effectively suppress both MAI and ISI without noise enhancement. The rationale is that the received signals in neighbouring time epochs are highly correlated after convolving with multipath channel. Therefore, the 
detected information bits in one time epoch can be used to improve the signal detection in the next time epoch, e.g., the interfering signals from the previous epochs are deducted from the received signal so that the quality of detection can be improved. The direct result is that the decorrelating detector operates in a rank-reduced signal subspace and the noise enhancement factor is reduced and keeps static over a wide range of multipath spreads and number of user. The performance gain is especially significant for systems with long delay spread and heavy loads. Meanwhile, the rank-reduced decorrelating detector has reduced complexity compared with the conventional expanded decorrelating detectors. And the decorrelating detector mitigates the error propagation problem caused by incorrect decision feedback. An iterative detection strategy can be further derived from this hybrid structure. In each iteration, by feeding back and forward the information from the decorrelating detector, all ISI can be deducted from the input signal so that the decorrelating detector operates in a signal subspace with further reduced rank. Performance gains are obtained compared to the non-recursive hybrid detector, but the improvement is not significant, since the latter has already approached the performance of the decorrelating detector in Gaussian channel. This novel hybrid detection structure which brings improved performance is the first contribution of this paper.

The second contribution is the development of a Kalman filter-based amplitude/power estimation algorithm. The estimated amplitudes are then used by the IC scheme. Kalman filter is well known in the optimal statistical estimation and control theory [27]. When the linear and Gaussian assumptions hold, no other algorithms can outperform Kalman filter [28]. Kalman filter has previously been used for blind and nonblind multiuser detection [14] [29]. In this paper, we show that Kalman filter and decorrelating detector can be perfectly integrated together to estimate the signal amplitudes for the purpose of interference cancellation. One of the desirable properties of the decorrelating detector is that it does not require any a priori information of the signal amplitudes (thus it is immune to the near-far effect). However, in this work, we take advantage of the fact that the output of the decorrelating detector actually contains rich amplitude information, and both soft and hard information can be utilized by the Kalman filter to estimate the amplitudes. The proposed adaptive amplitude estimation algorithm converges fast and tracks accurately. 
The third contribution of this paper is to develop a remedy algorithm to deal with the subspace collapse problem, which could happen to the expanded decorrelating detectors when the subspace rank is deficient under certain channel conditions. This problem can severely deteriorate the performance of the detector. The proposed remedy algorithm detects the rank deficiency and prevents malfunction in the expanded decorrelating detector.

This paper is focused on the synchronous multipath channel. However, asynchronous multipath channels can be modelled similarly as the one of synchronous channels. The only difference between them is the time shift of the interfering signals (both ISI and MAI) according to the relative delay of each user. With the known or estimated delay information, the proposed hybrid detection strategy can be easily extended to asynchronous channels.

\section{Signal Model for Multipath Synchronous Channel}

We consider a synchronous $K$-user direct-sequence (DS) CDMA system employing binary phase-shift keying (BPSK) modulation to transmit signal over multipath frequency-selective fading channels, as illustrated in Figure 1. The signal transmitted by the $k$ th user can be written as

$$
x_{k}(t)=\sum_{i=-\infty}^{\infty} A_{k} b_{k}(i) c_{k}(t-i T)
$$

where $A_{k}, b_{k}(i)$ and $c_{k}(t)$ are the amplitude, $i$ th transmitted information bit, and normalized signature waveform of the $k$ th user, respectively; $T$ is the symbol interval. $b_{k}(i) \in\{+1,-1\}$ follows identical and independent distribution (i.i.d.), and $c_{k}(t)$ is defined in [0,T]. In DS-CDMA systems, $c_{k}(t)$ is of the form

$$
c_{k}(t)=\sum_{n=0}^{N-1} c_{k}(n) \psi\left(t-n T_{c}\right), \quad t \in[0, T], k=1, \ldots, K
$$

where $N$ is the spreading gain; $\left\{c_{k}(n)\right\}_{n=0}^{N-1}$ is the real-valued and normalized signature code assigned to the $k$ th user, $\sum_{n=0}^{N-1} c_{k}^{2}(n)=1 ; T_{c}$ is the chip interval, $N T_{c}=T ; \psi(t)$ is a normalized chip waveform defined in $\left[0, T_{c}\right], \int_{0}^{T_{c}} \psi^{2}(t) d t=1$

In the case when a spreading sequence bandwidth $W$ is much larger than the coherence channel 
bandwidth, the tapped delay line model is widely used for multipath frequency-selective channels (Chap.4, [14]). In this work, we adopt a tapped delay line model with tap spacing $1 / W$ and tap coefficients $\left\{h_{k}(l)\right\}_{l=0}^{L-1}$, where $L$ is the number of resolvable paths for each user. We consider the case where $W=1 / T_{c}, L=\left\lceil T_{m} / T_{c}\right\rceil$, and $T_{m}$ is the multipath delay spread. In literature, it is usually assumed that $L<N$, i.e., the multipath spread is less than one symbol duration. In this paper, we consider a more general and realistic scenario in high-speed CDMA communications where the delay spread can be arbitrarily long. The impulse response of the multipath channel is modeled as

$$
h_{k}(t)=\sum_{l=0}^{L-1} h_{k}(l) \delta\left(t-\frac{l}{W}\right)
$$

where $\delta(t)$ is the Dirac's delta function. Transmitting the signals over the multipath channel expressed by (3), the received signal due to the $k$ th user is given by

$$
y_{k}(t)=x_{k}(t) * h_{k}(t)=\sum_{i=-\infty}^{\infty} A_{k} b_{k}(i) \sum_{l=0}^{L-1} h_{k}(l) c_{k}\left(t-i T-\frac{l}{W}\right)
$$

where '*' denotes the convolution operation. Finally, the total received signal is the superposition of $\mathrm{K}$ users' information-bearing data signals plus the AWGN, and can be expressed as

$$
r(t)=\sum_{k=1}^{K} y_{k}(t)+v(t)
$$

where $v(t)$ is a zero-mean white Gaussian noise. For simplicity, synchronous channel is the focus of this paper, however, the proposed scheme can be readily extended to asynchronous channels.

The received signal is filtered by a chip-matched filter and then sampled at chip rate, resulting in a discrete-time format of the signal model, in which the signal component due to the $p$ th chip of the $m$ th symbol from the $k$ th user is given by

$$
\begin{aligned}
y_{k}(m, p) & =\int_{m T+p T_{c}}^{m T+(p+1) T_{c}} y_{k}(t) \psi\left(t-m T-p T_{c}\right) d t \\
& =\sum_{i=-\infty}^{\infty} A_{k} b_{k}(i) \sum_{l=0}^{L-1} h_{k}(l) \sum_{n=0}^{N-1} c_{k}(n) \int_{m T+p T_{c}}^{m T+(p+1) T_{c}} \psi\left(t-i T-l T_{c}-n T_{c}\right) \psi\left(t-m T-p T_{c}\right) d t \\
& =\sum_{i=-\infty}^{\infty} A_{k} b_{k}(i) \sum_{l=0}^{L-1} h_{k}(l) \sum_{n=0}^{N-1} c_{k}(n) \int_{0}^{T_{c}} \psi\left[t+(m-i) T+(p-l-n) T_{c}\right] \psi(t) d t \\
& =\sum_{i=-\infty}^{\infty} A_{k} b_{k}(i) \sum_{l=0}^{L-1} h_{k}(l) c_{k}(p+m N-l-i N)
\end{aligned}
$$


Define $j=p+m N, y_{k}(j)=y_{k}(m, p)$, equation (6) can be rewritten as

$$
y_{k}(j)=\sum_{i=-\infty}^{\infty} A_{k} b_{k}(i) \sum_{l=0}^{L-1} h_{k}(l) c_{k}(j-l-i N)=\sum_{i=-\infty}^{\infty} A_{k} b_{k}(i) s_{k}(j-i N)
$$

where

$$
s_{k}(n) \stackrel{\Delta}{=} \sum_{l=0}^{L-1} h_{k}(l) c_{k}(n-l)
$$

Comparing (7) with (1), one can see that the received signature code is the distorted version of the original signature code after convolving with the multipath channel. Finally, the total received signal is expressed in discrete-time format as

$$
r(j)=\sum_{k=1}^{K} y_{k}(j)+v(j)
$$

where $v(j)=\int_{j T_{c}}^{(j+1) T_{c}} v(t) \psi\left(t-j T_{c}\right) d t$

At the output of the transmitter, the energy of each symbol is limited to duration $N T_{c}$. However, a close examination at (8) reveals that in the received signal, the energy of each symbol has been spread over an extended interval $(N+L-1) T_{c}$ due to the channel convolution, and hence the original spreading sequence $\left\{c_{k}(n)\right\}_{n=0}^{N-1}$ of the length $N$ becomes the actual received signature sequence $\left\{s_{k}(n)\right\}_{n=0}^{N+L-2}$ of length $N+L-1$. Naturally, we employ an $(N+L-1)$-observation window as illustrated in Figure 2. Note that this diagram just shows a special case when $N<L<2 N$, but in the theoretical derivation, we do not impose any constraint on the value of $L$. To detect each information bit, $N+L-1$ samples are stacked to form the received signal vector, and $L-1$ samples of them can be reused to detect the next bit, leading to some saving in sampling time and memory. Falling within the observation window are not only the information bits at the current time instant, but also those from the preceded instants and the succeeded instants. The received signal vector therefore contains intersymbol interference from the past as well as from the future symbols. To state this problem more clearly, we define $P \stackrel{\text { def }}{=}\lceil(L-1) / N\rceil$. Then, in the processing window of length $N+L-1,2 P+1$ symbols of the desired user are involved, including the current symbol, $P$ symbols before and $P$ symbols after the current symbol. Taking account of all the $K$ users, there are $(2 P+1) K$ symbols involved in detecting 
each symbol from the desired user. To summarize, the $(N+L-1) \times 1$-dimensional received signal vector for this system can be expressed as

$$
\begin{aligned}
\mathbf{r}(i) & =\sum_{k=1}^{K} \mathbf{y}_{k}(i)+\mathbf{v}(i) \\
& =\sum_{k=1}^{K}\left[A_{k} b_{k}(i) \mathbf{S}_{k}+\sum_{j=1}^{P} A_{k} b_{k}(i-j) \overline{\mathbf{s}}_{k, j}+\sum_{j=1}^{P} A_{k} b_{k}(i+j) \overline{\overline{\mathbf{s}}}_{k, j}\right]+\mathbf{v}(i)
\end{aligned}
$$

where

$$
\begin{aligned}
& \mathbf{y}_{k}(i)=A_{k} b_{k}(i) \mathbf{s}_{k}+\sum_{j=1}^{P} A_{k} b_{k}(i-j) \overline{\mathbf{s}}_{k, j}+\sum_{j=1}^{P} A_{k} b_{k}(i+j) \overline{\overline{\mathbf{s}}}_{k, j} \\
& \mathbf{s}_{k}=\left[\begin{array}{lll}
s_{k}(0) & \ldots & s_{k}(N+L-2)
\end{array}\right]^{T} \\
& \overline{\mathbf{s}}_{k, j}=\left[\begin{array}{lllllll}
s_{k}(j N) & s_{k}(j N+1) & \ldots & s_{k}(N+L-2) & 0 & \ldots & 0
\end{array}\right]^{T}, j=1 \ldots P \\
& \overline{\overline{\mathbf{s}}}_{k, j}=\left[\begin{array}{lllllll}
0 & \ldots & 0 & s_{k}(0) & s_{k}(1) & \ldots & s_{k}(N+L-2-j N)
\end{array}\right]^{T}, j=1 \ldots P \\
& \mathbf{v}(i)=\left[\begin{array}{lll}
v(i N) & \ldots & v(i N+N+L-2)
\end{array}\right]^{T}
\end{aligned}
$$

Each element of the $(N+L-1) \times 1$-dimensional noise vector $\mathbf{v}(i)$ has variance $\sigma^{2} \mathbf{I}$. When $L=1$ (hence $P=0$ ) and $h_{k}(1)=1, k=1 \ldots K, \overline{\mathbf{s}}_{k, j}$ in (13) and $\overline{\overline{\mathbf{s}}}_{k, j}$ in (14) will be zero according to the definition of $s_{k}(n)$ in (8), and (10) is simplified to the model for the single-path flat fading channel

$$
\mathbf{r}(i)=\sum_{k=1}^{K} A_{k} b_{k}(i) \mathbf{c}_{k}+\mathbf{v}(i)
$$

Defining

$$
\begin{aligned}
& \overline{\mathbf{s}}_{k}=\left[\begin{array}{lll}
\overline{\mathbf{s}}_{k, 1} & \ldots & \overline{\mathbf{s}}_{k, P}
\end{array}\right]_{(N+L-1) \times P} \\
& \overline{\overline{\mathbf{s}}}_{k}=\left[\begin{array}{lll}
\overline{\overline{\mathbf{s}}}_{k, 1} & \ldots & \overline{\mathbf{s}}_{k, P}
\end{array}\right]_{(N+L-1) \times P} \\
& \mathbf{S}=\left[\begin{array}{lllllll}
\mathbf{s}_{1} & \overline{\mathbf{s}}_{1} & \overline{\mathbf{s}}_{1} & \ldots & \mathbf{s}_{K} & \overline{\mathbf{s}}_{K} & \overline{\overline{\mathbf{s}}}_{K}
\end{array}\right]_{(N+L-1) \times(2 P+1) K}
\end{aligned}
$$

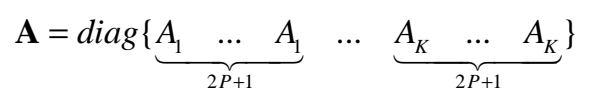

$$
\begin{aligned}
& \mathbf{b}_{k}(i)=\left[b_{k}(i) \quad b_{k}(i-1) \quad \ldots \quad b_{k}(i-P) \quad b_{k}(i+1) \quad \ldots \quad b_{k}(i+P)\right]_{1 \times(2 P+1)}, k=1 \ldots K \\
& \mathbf{b}(i)=\left[\begin{array}{lll}
\mathbf{b}_{1}(i) & \ldots & \mathbf{b}_{K}(i)
\end{array}\right]^{T}
\end{aligned}
$$


then (10) can be written in a more compact vector/matrix format as

$$
\mathbf{r}(i)=\mathbf{S A b}(i)+\mathbf{v}(i)
$$

Assume that $\left\{\mathbf{s}_{k}\right\}_{k=1}^{K},\left\{\overline{\mathbf{S}}_{k}\right\}_{k=1}^{K}$ and $\left\{\overline{\overline{\mathbf{s}}}_{k}\right\}_{k=1}^{K}$ are independent vectors, then the column vectors of $\mathbf{S}$ spans the signal subspace $\mathfrak{R}^{(N+L-1) \times(2 P+1) K}$. Compared with the AWGN channel in which the signal subspace is $\mathfrak{R}^{N \times K}$, the rank of the signal subspace for the multipath channel is $2 P K$ higher. For the signal model in (21), the linear multiuser detection for the $k$ th user is to do correlation and then take hard decision, i.e.,

$$
\hat{b}_{k}(i)=\operatorname{sgn}\left(\mathbf{d}_{k}^{T} \mathbf{r}(i)\right)
$$

where $\mathbf{d}_{k} \in \mathfrak{R}^{N+L-1}$. Similar to [26], three linear multiuser detectors can be obtained as follows:

\section{1) Linear Expanded Decorrelating Detector}

Assume that all users' spreading codes are known at the receiver, the expanded decorrelating detector for the first user is given by

$$
\mathbf{d}_{1}=\mathbf{S}\left[\mathbf{M}^{-1}\right]_{\bullet, 1}
$$

where $\left[\mathbf{M}^{-1}\right]_{\bullet, 1}$ denotes the $k$ th column of the matrix $\mathbf{M}^{-1}$, and

$$
\mathbf{M}=\mathbf{S}^{T} \mathbf{S}
$$

$\mathbf{d}_{1}$ is a $(N+L-1)$-vector. It completely removes MAI and ISI.

\section{2) Expanded Subspace-Based Decorrelating Detector}

In [11], blind multiuser detectors are expressed in the closed format of subspace components without need of the interfering users' spreading codes. The expanded subspace-based decorrelating detector in this subsection and the expanded subspace-based MMSE detector in the next subsection are the extension of them from the AWGN channel to multipath channels. In this paper, we assume that channels are known; hence the expanded subspace-based detectors are not blind. The eigenvectors and eigenvalues of the signal subspace can be obtained, e.g., by conducting eigendecomposition on the autocorrelation matrix $E\left\{\mathbf{r r}^{T}\right\}$. The decorrelating detector is given by

$$
\mathbf{d}_{1}=\mathbf{U}_{s} \boldsymbol{\Lambda}_{s}^{-1} \mathbf{U}_{s}^{T} \mathbf{S}_{1} /\left[\mathbf{s}_{1}^{T} \mathbf{U}_{s} \boldsymbol{\Lambda}_{s}^{-1} \mathbf{U}_{s}^{T} \mathbf{s}_{1}\right]
$$


where $\mathbf{U}_{s}$ is $(N+L-1) \times(2 P+1) K$ matrix which columns are the eigenvectors for the signal subspace, and $\boldsymbol{\Lambda}_{s}$ is a $(2 P+1) K \times(2 P+1) K$ diagonal matrix which diagonal elements are the corresponding eigenvalues.

\section{3) Expanded Subspace-Based MMSE Detector}

The MMSE detector expressed in closed form of the expanded signal subspace components is given by

$$
\mathbf{d}_{1}=\mathbf{U}_{s}\left(\boldsymbol{\Lambda}_{s}+\sigma^{2} \mathbf{I}_{s}\right)^{-1} \mathbf{U}_{s}^{T} \mathbf{s}_{1} /\left[\mathbf{s}_{1}^{T} \mathbf{U}_{s}\left(\boldsymbol{\Lambda}_{s}+\sigma^{2} \mathbf{I}_{s}\right)^{-1} \mathbf{U}_{s}^{T} \mathbf{s}_{1}\right]
$$

where $\mathbf{I}_{s}$ is a $(2 P+1) K \times(2 P+1) K$ identity matrix.

Discussion:

1. Given that the estimated eigencomponents in (25) are accurate, the decorrelating detectors in (23) and (25) give identical performance because they are the same detector of different expressions. Both suffer the noise enhancement problem. The noise is enhanced more when the subspace rank increases, as will be shown by simulation. The increase in subspace rank can be result of the increasing number of users or longer channel spread.

\section{The Hybrid Multiuser Detector}

In this section, we develop a hybrid multiuser detector which is designed to tackle the noise enhancement problem inherent to the decorrelating detectors introduced in the previous section. The structure of the proposed receiver is illustrated in Figure 3. The received signal $\mathbf{r}(i)$ is pre-processed by subtracting the interfering signal $\hat{\mathbf{I}}(i-)$ which is contributed by the symbols in the previous epochs, and then the resulting signal $\tilde{\mathbf{r}}(i)$ is fed into the rank-reduced expanded decorrelating detector. Hard decision of the decorrelating detector forms the estimate of the symbol bits $\hat{\mathbf{b}}(i)$. Both the soft decision and the hard decision $\hat{\mathbf{b}}(i)$ are sent into the Kalman filter bank to track the signal amplitudes of all users. Finally the estimated amplitudes $\left\{\hat{A}_{k}(i)\right\}_{k=1}^{K}$ and the estimated bits $\hat{\mathbf{b}}(i)$ are sent to the ISI synthesiser to recreate the interference signals, which will be used by the interference canceller in preprocessing the next received signal. 


\section{A. Interference Synthesis and Cancellation}

The item $\sum_{k=1}^{K} \sum_{j=1}^{P} A_{k} b_{k}(i-j) \overline{\mathbf{s}}_{k, j}$ in (10) is due to the interfering signals from the previous epochs. They can be recreated by the ISI synthesiser at time $i$ because the bits before time $i$ have already been detected. With the estimated amplitudes $\left\{\hat{A}_{k}(i-1)\right\}_{k=1}^{K}$ and the detected bits $\left\{\hat{b}_{k}(i-j)\right\}_{j=1 \ldots P, k=1 \ldots K}$, the interference due to the previous symbols are recreated as

$$
\hat{\mathbf{I}}(i-)=\sum_{k=1}^{K} \sum_{j=1}^{P} \hat{A}_{k}(i-1) \hat{b}_{k}(i-j) \overline{\mathbf{s}}_{k, j}
$$

Interference cancellation results in the deflated received signal as given by

$$
\begin{aligned}
\tilde{\mathbf{r}}(i) & =\mathbf{r}(i)-\hat{\mathbf{I}}(i-) \\
& =\sum_{k=1}^{K}\left[A_{k} b_{k}(i) \mathbf{s}_{k}+\sum_{j=1}^{P}\left[A_{k} b_{k}(i-j)-\hat{A}_{k}(i-1) \hat{b}_{k}(i-j)\right] \overline{\mathbf{s}}_{k, j}+\sum_{j=1}^{P} A_{k} b_{k}(i+j) \overline{\overline{\mathbf{s}}}_{k, j}\right]+\mathbf{v}(i) \\
& \approx \sum_{k=1}^{K}\left[A_{k} b_{k}(i) \mathbf{s}_{k}+\sum_{j=1}^{P} A_{k} b_{k}(i+j) \overline{\overline{\mathbf{s}}}_{k, j}\right]+\mathbf{v}(i)
\end{aligned}
$$

The approximation in (28) is valid when the adaptive estimations of $\hat{A}_{k}(i-1)$ and $\hat{\mathbf{b}}_{k}(i-j)$ converge.

\section{B. Rank-Reduced Expanded Decorrelating Detector}

The rank of the original expanded signal subspace for $(10)$ is $(2 P+1) K$. The interference cancellation reduces the subspace rank by $P K$. Hence the remaining rank for the deflated signal in (28) is $(P+1) K$. Define

$$
\tilde{\mathbf{S}}=\left[\begin{array}{lllll}
\mathbf{s}_{1} & \overline{\mathbf{s}_{1}} & \ldots & \mathbf{s}_{K} & \overline{\mathbf{s}}_{K}
\end{array}\right]_{(N+L-1) \times(P+1) K}
$$

The rank-reduced expanded decorrelating detector for the $k$ th user is

$$
\tilde{\mathbf{d}}_{k}=\tilde{\mathbf{S}}\left[\tilde{\mathbf{M}}^{-1}\right]_{\cdot, k}
$$

where

$$
\tilde{\mathbf{M}}=\tilde{\mathbf{S}}^{T} \tilde{\mathbf{S}}
$$

\section{Amplitude Estimation Using Kalman Filter}

In stationary or slowly-time varying system, the signal amplitudes do not change within one symbol duration. Thus, for the $k$ th user, the state transition equation is given by 


$$
A_{k}(i)=A_{k}(i-1)
$$

The output of the $k$ th user's decorrelating detector is

$$
z_{k}(i)=\tilde{\mathbf{d}}_{k}^{T} \tilde{\mathbf{r}}(i)=b_{k}(i) A_{k}(i)+\tilde{\mathbf{d}}_{k}^{T} \mathbf{v}(i)
$$

Equation (33) is the measurement equation because it relates the state variable $A_{k}(i)$ to the measurement $z_{k}(i)$. The measurement matrix is $b_{k}(i)$ which is time-varying (here it is a scalar and takes value in $\{+1,-1\}$ ). Obviously, the mean and covariance of the process noise in (32) are zeros. The measurement noise in (33) is $\tilde{\mathbf{d}}_{k}^{T} \mathbf{v}(i)$ with mean and covariance

$$
\begin{gathered}
E\left\{\tilde{\mathbf{d}}_{k}^{T} \mathbf{v}(i)\right\}=\tilde{\mathbf{d}}_{k}^{T} E\{\mathbf{v}(i)\}=0 \\
\xi=\operatorname{cov}\left\{\tilde{\mathbf{d}}_{k}^{T} \mathbf{v}(i)\right\}=\tilde{\mathbf{d}}_{k}^{T} E\left\{\mathbf{v}(i) \mathbf{v}(i)^{T}\right\} \tilde{\mathbf{d}}_{k}=\sigma^{2} \tilde{\mathbf{d}}_{k}^{T} \tilde{\mathbf{d}}_{k}
\end{gathered}
$$

Replacing $b_{k}(i)$ in (33) with its estimation

$$
\hat{b}_{k}(i)=\operatorname{sgn}\left(z_{k}(i)\right)
$$

the measurement equation is reformed as

$$
z_{k}(i)=\hat{b}_{k}(i) A_{k}(i)+\tilde{\mathbf{d}}_{k}^{T} \mathbf{v}(i)
$$

Based on (32) and (37), the resulting Kalman filtering estimation routine is summarized in Table 1. The computational complexity including interference cancellation and Kalman filtering is $O\left(P^{2} K N\right)$.

Table 1: Amplitude estimation algoritmm using Kalman filter

/* Initialization: */

$A_{k}(0)=0, P(0)=1$

/*stimation */

FOR $\quad i=1,2, \ldots$

$$
\begin{aligned}
& z_{k}(i)=\mathbf{d}_{k}^{T} \tilde{\mathbf{r}}(i) \\
& \hat{b}_{k}(i)=\operatorname{sgn}\left(z_{k}(i)\right) \\
& K(i)=P(i-1) \hat{b}_{k}(i)(P(i-1)+\xi)^{-1}
\end{aligned}
$$




$$
\begin{aligned}
& P(i)=\left(1-K(i) \hat{b}_{k}(i)\right) P(i-1) \\
& A_{k}(i)=A_{k}(i-1)+K(i)\left(z_{k}(i)-\hat{b}_{k}(i) A_{k}(i-1)\right)
\end{aligned}
$$

\section{Iterative Multiuser Detection}

In Figure 3, only the intersymbol interference from the previous epochs is cancelled. In the first detection iteration, the ISI due to the symbols of the future epochs are not known and hence cannot be cancelled. After the whole frame of signals have been detected, the estimated information bits can be fed back, and then the interference due to the future epochs can be cancelled in the same way as the previous epochs. The rank of the resulting signal subspace will be further reduced to $K$. The interference cancellation and decorrelating detection procedures can be operated on the same signal frame repeatedly, and the performance of the detection can be improved in an iterative manner. Figure 4 illustrates this idea. The deflated received signal becomes

$$
\tilde{\mathbf{r}}(i)=\mathbf{r}(i)-\hat{\mathbf{I}}(i-)-\hat{\mathbf{I}}(i+)
$$

where $\hat{\mathbf{I}}(i+)$ is the recreated interfering signals due to the future epochs, and is defined as

$$
\hat{\mathbf{I}}(i+)=\sum_{k=1}^{K} \sum_{j=1}^{P} \hat{A}_{k}(i-1) \hat{b}_{k}(i+j) \overline{\overline{\mathbf{S}}}_{k, j}
$$

For the iterative detection, (29) is changed to

$$
\tilde{\tilde{\mathbf{S}}}=\left[\begin{array}{llll}
\mathbf{s}_{1} & \mathbf{s}_{2} & \ldots & \mathbf{s}_{K}
\end{array}\right]_{(N+L-1) \times K}
$$

The rank-reduced decorrelating detector for the $k$ th user is

$$
\tilde{\mathbf{d}}_{k}=\tilde{\tilde{\mathbf{S}}}\left[\tilde{\tilde{\mathbf{M}}}^{-1}\right]_{\bullet, k}
$$

where

$$
\tilde{\tilde{\mathbf{M}}}=\tilde{\tilde{\mathbf{S}}}^{T} \tilde{\tilde{\mathbf{S}}}
$$




\section{Subspace Collapse and Remedy Algorithm}

In computing the expanded decorrelating detectors in (23) and (30), we have assumed that all column vectors of $\mathbf{S}$ (see (17)) and $\tilde{\mathbf{S}}$ (see (29)) are linearly independent. However, this is not always guaranteed in practice. As an example, for zero-padded $\overline{\mathbf{s}}_{k, p}$ and $\overline{\overline{\mathbf{s}}}_{k, p}, k=1, \ldots, K$ and $p=1, \ldots, P$, if $L-1=(P-1) N+1$, then $\overline{\mathbf{s}}_{1, P}=\left[\begin{array}{lllll}s_{1}(P N+1) & 0 & \ldots & 0\end{array}\right]^{T}, \ldots, \quad \overline{\mathbf{s}}_{K, P}=\left[\begin{array}{lllll}s_{K}(P N+1) & 0 & \ldots & 0\end{array}\right]^{T}$ are correlated; and $\overline{\overline{\mathbf{s}}}_{1, P}=\left[\begin{array}{llll}0 & 0 & \ldots & s_{1}(0)\end{array}\right]^{T}, \ldots, \overline{\overline{\mathbf{s}}}_{K, P}=\left[\begin{array}{llll}0 & 0 & \ldots & s_{K}(0)\end{array}\right]^{T}$ are also correlated. The column vectors of $\mathbf{S}$ do not span subspace $\Re^{(2 P+1) K \times(N+L-1)}$, and the column vectors of $\tilde{\mathbf{S}}$ do not span subspace $\mathfrak{R}^{(P+1) K \times(N+L-1)}$. We say that the expanded signal subspace has collapsed. The expanded decorrelating detectors in (23) and (30) are no longer valid as an immediate consequence of subspace collapse. In order to tackle this problem, we propose a remedy algorithm as outlined in Table 2 to detect the subspace collapse and then compute the expanded detectors in the new subspace. The method is to remove the column vectors that are not independent. Because column vectors in $\left\{\mathbf{s}_{k}\right\}_{k=1}^{K}$ have the full energy of the current symbols and they are usually linearly independent, the removing operation will only be carried out to the vectors $\left\{\overline{\mathbf{s}}_{k, \tau}\right\}_{\tau=1}^{P}$ and $\left\{\overline{\overline{\mathbf{s}}}_{k, \tau}\right\}_{\tau=1}^{P}, k=1 \ldots K$. Since the methods are the similar for the expanded detector in (23) and the rank-reduced expanded detector in (30), we only outline the algorithm for the rank-reduced expanded detector as in Table 2.

Table 2: Remedy Algorithm for the Rank-Reduced Expanded Decorrelating Detector

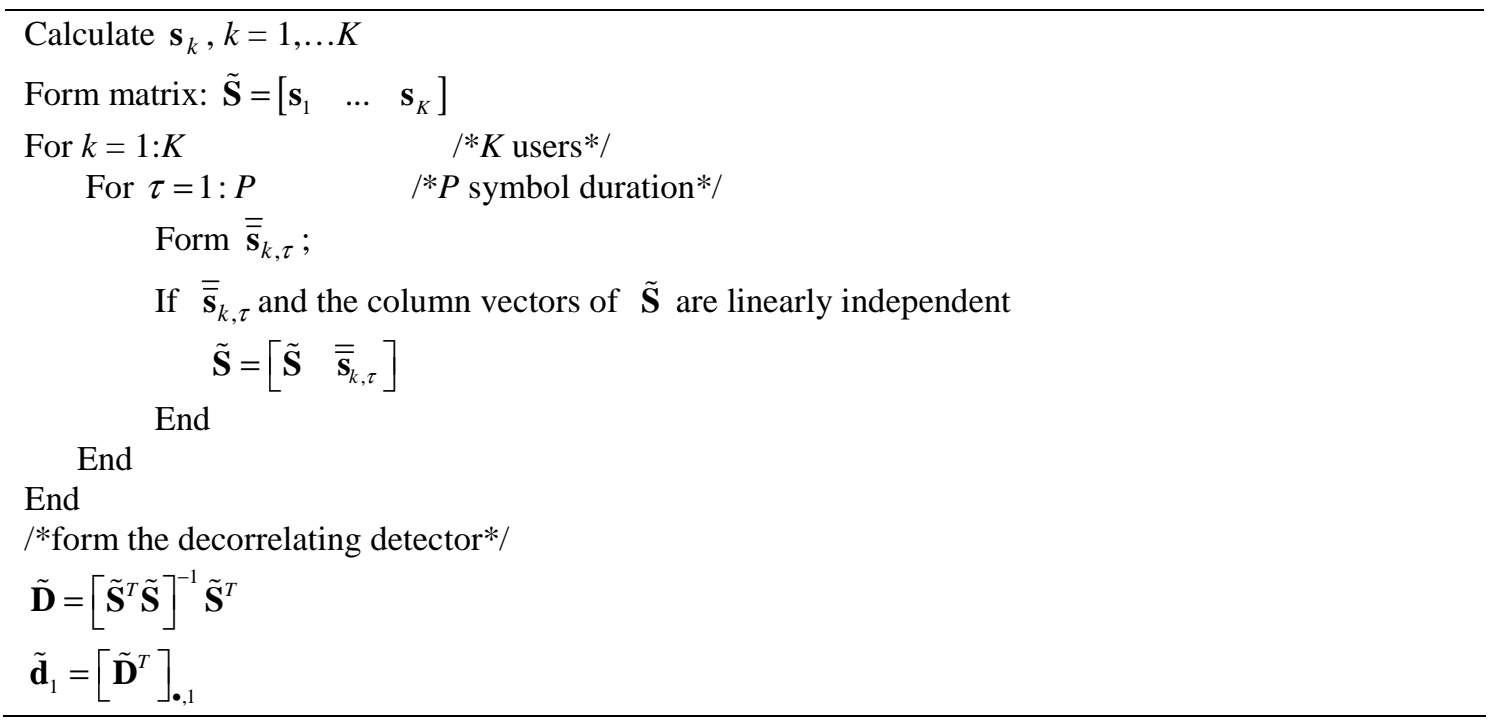




\section{Performance Analysis}

Signal-to-interference-plus-noise ratio (SINR) in the output of the linear detector for the $k$ th user at time $i$ is defined as

$$
\operatorname{SINR}(i)=\frac{E\left\{\left[\mathbf{d}_{k}^{T} A_{k} b_{k}(i) \mathbf{s}_{k}\right]^{2}\right\}}{E\left\{\left[\mathbf{d}_{k}^{T}\left(\mathbf{r}(i)-A_{k} b_{k}(i) \mathbf{s}_{k}\right)\right]^{2}\right\}}
$$

For the full-rank expanded decorrelating detector,

$$
\begin{gathered}
\mathbf{d}_{k}^{T} \mathbf{s}_{k}=1 \\
\mathbf{d}_{k}^{T} \mathbf{s}_{l}=0, l \neq k \\
\mathbf{d}_{k}^{T} \overline{\mathbf{s}}_{l}=0, \forall l \\
\mathbf{d}_{k}^{T} \overline{\overline{\mathbf{s}}_{l}}=0, \forall l
\end{gathered}
$$

so the SINR in its output is

$$
\operatorname{SINR}_{\text {full }}(i)=\frac{A_{k}^{2}}{\mathbf{d}_{k}^{T} \mathbf{d}_{k} \sigma^{2}}=\frac{A_{k}^{2}}{\alpha_{k} \sigma^{2}}
$$

where $\alpha_{k}=\mathbf{d}_{k}^{T} \mathbf{d}_{k}$ is the noise enhancement factor. Compared with the SNR in the received signal which is $A_{k}^{2} / \sigma^{2}$, SINR in (52) is reduced by $\alpha_{k}$ times, or equally, the noise is amplified by $\alpha_{k}$ times. To gain more insight of the structure of the noise enhancement factor, it can be expressed as

$$
\begin{aligned}
\alpha_{k} & =\left[\mathbf{M}^{-1}\right]_{k, \bullet} \mathbf{S}^{T} \mathbf{S}\left[\mathbf{M}^{-1}\right]_{\bullet, k} \\
& =\left[\mathbf{M}^{-1}\right]_{k, \bullet} \mathbf{M}\left[\mathbf{M}^{-1}\right]_{\bullet, k} \\
& =\left[\left(\mathbf{S}^{T} \mathbf{S}\right)^{-1}\right]_{k, k}
\end{aligned}
$$

It equals the $k$ th main diagonal element of the matrix $\left(\mathbf{S}^{T} \mathbf{S}\right)^{-1}$. Expressing (52) in $\log$ format shows the connection between the output SINR and the SNR in the received signal as follows

$$
\operatorname{SINR}_{\text {full }}(i)=\log _{10} \frac{A_{k}^{2}}{\sigma^{2}}-\log _{10} \alpha_{k}=\operatorname{SNR}-\log _{10} \alpha_{k} \quad(d B)
$$

For the rank-reduced expanded decorrelating detector, the interference that is not completely cancelled can be modelled as Gaussian noise with variance $\sigma_{I}^{2}$. Then the SINR is given by 


$$
\operatorname{SINR}_{\text {reduce }}(i)=\frac{A_{k}^{2}}{\tilde{\mathbf{d}}_{k}^{T} \tilde{\mathbf{d}}_{k}\left(\sigma^{2}+\sigma_{I}^{2}\right)}=\frac{A_{k}^{2}}{\tilde{\alpha}_{k}\left(\sigma^{2}+\sigma_{I}^{2}\right)}
$$

where $\tilde{\alpha}_{k}=\tilde{\mathbf{d}}_{k}^{T} \tilde{\mathbf{d}}_{k}$, and similarly it has structure

$$
\tilde{\alpha}_{k}=\left[\left(\tilde{\mathbf{S}}^{T} \tilde{\mathbf{S}}\right)^{-1}\right]_{k, k}
$$

After the Kalman filter converges, $\hat{\mathbf{I}}(i-)$ is almost equal to $\sum_{k=1}^{K} \sum_{j=1}^{P} A_{k}(i-1) b_{k}(i-j) \overline{\mathbf{s}}_{k, j}$, and $\sigma_{I}^{2} \approx 0$, then the $\log$ format of (55) is given by

$$
\operatorname{SINR}_{\text {reduce }}(i)=\operatorname{SNR}-\log _{10} \tilde{\alpha}_{k} \quad(d B)
$$

After convergence, the SINR improvement achieved by the proposed hybrid detector over the full-rank expanded decorrelating detector is given by

$$
\Delta \operatorname{SINR}(i)=\operatorname{SINR}_{\text {reduce }}(i)-\operatorname{SINR}_{\text {full }}(i)=\log _{10} \alpha_{k}-\log _{10} \tilde{\alpha}_{k} \quad(d B)
$$

Equation (58) shows that the performance improvement is decided by the decorrelating detector and is independent of the input SNR.

\section{Numerical Results}

The proposed algorithms are evaluated by computer simulation in this section. Assume that there are 10 users in the synchronous DS-CDMA system unless otherwise stated $(K=10)$. User 1 is the interested user. Users' transmission power is unbalanced: compared with user 1, users 2-6 are 10dB stronger, users $7-9$ are $20 \mathrm{~dB}$ stronger and user 10 is $30 \mathrm{~dB}$ stronger, respectively. The spreading codes are randomly-generated, independent and normalized sequences and the spreading gain is $N=31$. The multipath channel coefficients are randomly-generated and normalized and have exponential decay profile. For the blind multiuser detectors, 5000 symbols are used to estimate signal subspace. All results are averaged over 500 Monte-Carlo runs in order to yield reliable statistics. SNR values are measured by comparing the power of user 1 and the background noise. In all simulations, the measurement noise variance $\xi$ in (42) is set to 0.1 .

Example 1: Amplitude tracking. Since amplitude estimation plays an important role in the proposed detection strategy, we study the effectiveness of the Kalman filter-based amplitude tracking algorithm in different scenarios. Figure 5 shows the amplitude tracking performance of all users when the 
multipath channel spread is 25 and the input SNR is $20 \mathrm{~dB}$. It shows that the adaptive process converges fast and the power estimation of all users is almost precise after about 50 iterations. The following examples are focused on the first user. In Figure 6, the multipath spread is 25 and the input SNR varies from $5 \mathrm{~dB}$ to $20 \mathrm{~dB}$. It can be seen that $\mathrm{SNR}$ at moderate or higher level is good for accurate power estimation, at lower SNR level, there is some loss in estimation accuracy (about 5\%). Figure 7 shows amplitude tracking when SNR is $20 \mathrm{~dB}$ and the multipath channel spreads vary from 25 , which is less than one symbol interval, to 86 , which is over two symbol intervals. It can be seen that the amplitude estimation algorithm is not significantly affected by the channel spreads.

Example 2: SINR performance. Figure 8 to Figure 10 compare the SINR performance of three multiuser detectors at different multipath channels and different input SNR levels. The investigated detectors are the expanded decorrelating detector (ExpLDD), the rank-reduced expanded decorrelating detector assisted by fed-forward interference cancellation (ExpLDD_IC1), and the rank-reduced expanded decorrelating detector assisted by fed-forward and fed-back interference cancellation (ExpLDD_IC2). The figures show that both rank-reduced expanded decorrelating detectors obtain dramatic performance improvement compared with the full-rank expanded decorrelating detector. For a given multipath channel, the SINR improvement is almost the same regardless of input SNR. This is understandable from (58): $\triangle S I N R$ is only decided by the enhancement factors $\alpha_{k}$ and $\tilde{\alpha}_{k}$. On the other hand, the difference in the SINR improvement is significant in different channel conditions-more improvement at longer channel spread, e.g., when channel spread is 25 which is within one symbol interval, the SINR performance gain is about $1.5 \mathrm{~dB}$; when channel spread is 56 which is over one symbol interval, the gain is about $6 \mathrm{~dB}$; when channel spread is 86 which is over two symbol intervals, the gain is about $12 \mathrm{~dB}$. This improvement comes from the fact that the SINR performance of the rank -reduced decorrelating detectors almost does not change with the channel delay spread while the SINR performance of the full-rank expanded decorrelating detector degrades significantly with the channel delay spread. It is also observed that ExpLDD_IC2 does not perform significantly better than ExpLDD_IC1. In the following experiments, only ExpLDD_IC1 will be studied.

More detailed study about the SINR-channel performance is presented in Figure 11. The ExpLDD and ExpLDD_IC1 are compared and the remedy algorithms are applied to them because the expanded 
subspace collapses at some channel spreads. The received SNR is $20 \mathrm{~dB}$, and the multipath channel spreads vary from 10 to 85 . The plotted SINR is the converged SINR at each channel spread. The SINR for the ExpLDD_IC1 remains relatively static when the channel spread elongates, while the SINR decrease in the ExpLDD shows staircase effect when the channel spread crosses symbol intervals. The transition between stairs shows that the subspace collapse happens when the channel spread is just over integral times of the symbol interval.

In Figure 8 to Figure 11, the effect of the channel on the SINR performance is due to the change in the noise enhancement factors. The channel condition affects the structure of the expanded signal subspace, which consequently affects the noise enhancement factors. For example, the rank of the full-rank signal subspace is $(2 P+1) K$ and the rank of the rank-reduced signal subspace is $(P+1) K$. When $P$ increases by 1 , the rank of the full-rank signal subspace increases by $2 K$ and the rank of the reduced signal subspace increases by $K$. Figure 12 shows the noise enhancement factors of ExpLDD and ExpLDD_IC1 at different channel spreads. It can be seen that the noise enhancement factor of ExpLDD_IC1 almost does not change as the channel spread increases, while the noise enhancement factor of ExpLDD increases in a staircase style. This coincides with the result in Figure 11. Note that the subspace rank is not only determined by $P$ but also $K$. Increasing $K$ could also affect the SINR performance of the ExpLDD and ExpLDD_IC1 detectors. Figure 13 verifies this idea where the channel delay spread is 56. It shows that the noise enhancement factor of ExpLDD_IC1 increases dramatically as the number of users increases, while the noise enhancement factor of ExpLDD almost does not change.

Example 3: BER performance. Figure 14 compares the bit error rate (BER) performance of several multiuser detectors at different SNR levels for the 10-user system. The compared detectors are the RAKE receiver, the expanded decorrelating detector (ExpLDD), the expanded subspace-based decorrelating detector based on (SubExpLDD), the expanded subspace-based MMSE detector (SubExpMMSE), ExpLDD_IC1 with Kalman filter-based amplitude estimator (ExpLDD_IC1 +estimated amplitudes), and ExpLDD_IC1 with known amplitude (ExpLDD_IC1+genie amplitudes). The channel spread is set to be 56 . It can be seen from the figure that the RAKE detector fails to operate. This is because that the RAKE receiver is only optimum in single-user multipath system, but not in multiuser multipath system. It suffers severely from MAI. The other schemes are capable of joint 
suppression of MAI and ISI. ExpLDD and SubExpLDD do not yield satisfactory performance due to the noise enhancement problem, which can be effectively mitigated by the SubExpMMSE detector and the proposed hybrid multiuser detector. The performance of SubExpMMSE is subject to the accurate knowledge of the subspace components and the noise. For the presented SubExpMMSE result, 10000 symbols are used to estimate the subspace components. Figure 14 shows that the proposed scheme is superior to the ExpMMSE detector, and a gap of $2 \mathrm{~dB}$ is observed for this 10-user system at target BER around $10^{-4}$. As will become evident in the following example, the performance gain achieved by the proposed scheme becomes more significant when the system is more heavily loaded and the channel becomes more dispersive. We also see that the proposed scheme with estimated amplitude yields almost identical performance to the one with perfect amplitude estimation, which means the Kalman filter can estimate the amplitude very accurately, and the impact of estimation error on the system performance is negligible.

Example 4: Capacity comparison. The system capacity, i.e., the number of users that can be supported by the system with acceptable bit error rate, is examined in Figure 15 for the ExpLDD, SubExpLDD, SubExpMMSE and the ExpLDD_IC1. The channel spread and SNR value are set to be 56 and $15 \mathrm{~dB}$, respectively. It is assumed that the transmission power of all the other users is $10 \mathrm{~dB}$ higher than the user of interest. It is apparent to see from the figure that the proposed scheme increases the system capacity significantly compared to the other detectors. For instance, with a target BER $=10^{-4}$, over 14 users can be supported by the proposed scheme, whereas only 5 users can be supported by ExpLDD and SubExpLDD and 8 users can be supported by SubExpMMSE. Compared to the other detectors, the proposed scheme is much less affected by the number of active users, which makes it more effective for heavily loaded systems. We have also measured the system capacity at different levels of channel spread. The conclusion is that the proposed hybrid multiuser detector is rather robust against the channel dispersion, whereas the performance of other detectors degrades significantly as the channel becomes more dispersive. The plot is omitted here to conserve space.

\section{Conclusion}

A novel hybrid multiuser detection strategy for multipath frequency-selective fading channels has been developed in this paper. It has an IC pre-processing unit and an expanded decorrelating detector in the 
back end. The IC unit plays a crucial role in alleviating the noise enhancement problem suffered by the expanded decorrelating detector. This is achieved by cancelling ISI and hence reducing the signal subspace rank. Unlike the conventional interference cancellation techniques, the proposed IC scheme does not suffer from the error propagation problem and it is near-far resistant. High SNR is not required either. The rank-reduced decorrelating detector enjoys the advantages of the full-rank expanded decorrelating detector, such as complete MAI and ISI suppression, and in the meantime, overcomes the noise enhancement problem. The noise enhancement factor of the rank-reduced decorrelating detector is minor compared to the full-rank decorrelating detector and it is almost irrelevant to the channel delay spread and the system loads. The proposed amplitude tracking algorithm converges quickly and tracks accurately in moderate or higher SNR conditions. Compared to existing methods, dramatic performance gain in terms of SINR, BER and capacity has been achieved with this new multiuser detection approach. The detection scheme is proposed for synchronous scenario, but it can be easily extended into asynchronous scenario provided that the delay is known or can be estimated. Combining this new multiuser detection method with advanced channel coding technique and deriving an iterative multiuser detection and decoding algorithm as well as the use of the amplitude estimation algorithm for power control purpose will be the future research topics for the authors. 


\section{REFERENCES}

1. M. Sirbu, E. Delfino and V. Koivunen, "A novel method for time delay acquisition in satellite navigation systems," ISSSTA2004, Sydney, Australia, pp.726-730, Aug. 2004.

2. http://standards.ieee.org/getieee802/802.11.html (accessed on 26/04/2007)

3. http://standards.ieee.org/getieee802/802.15.html (accessed on 26/04/2007)

4. S. Verdu, Multiuser Detection, Cambridge University Press, 2001

5. S. Verdu, Optimum multiuser signal detection. $\mathrm{PhD}$ thesis, University of Illinois at UrbanaChampaign, Aug. 1986.

6. R. Lupas and S. Verdu, "Linear multiuser detector for synchronous code-division multipleaccess channels," IEEE Trans. Inform. Theory, vol. 35, pp. 123-136, 1989.

7. R. Lupas and S. Verdu, "Near-far resistance of multiuser detectors in asynchronous channels," IEEE Trans Commun., vol.38, pp496-508, 1990.

8. Z. Xie, R.T. Short and C.K. Rushforth, "A family of suboptimum detectors for coherent multiuser communications," IEEE J. Select. Areas Commun., vol.8, no. 4, pp.683 - 690, 1990.

9. U. Madhow, M. L. Honig, "MMSE interference suppression for direct-sequence spreadspectrum CDMA,” IEEE Trans. Commun., vol. 42, no. 12, pp. 3178-3188, 1994.

10. M.L. Honig, U. Madhow, S. Verdu, "Blind adaptive multiuser detection," IEEE Trans. Information Theory, vol. 41, no. 4, pp. 944-960, July 1995.

11. X. Wang and H.V. Poor, "Blind Multiuser Detection: A Subspace Approach," IEEE Trans. Information Theory, vol. 44, no.2, pp.677-690, 1998.

12. Chkeif, K. Abed-Meraim, G. Kawas-Kaleh, Y. Hua. "Spatio-temporal blind adaptive multiuser detection”. IEEE Transactions on Commun., vol. 48, no. 5, pp. 729-732, May 2000.

13. S. Roy: "Subspace blind adaptive detection for multiuser CDMA," IEEE Trans. Commun., vol.48, No.1, pp.169-175, Jan, 2000.

14. H. Zhou, W.L. Woo and B.S. Sharif, "Subspace-Based Blind Adaptive Multiuser Detection using Kalman Filter," IEE Proceedings Commun., vol.152, no.3, pp.302-310, June 2005.

15. M. Varanasi, B. Aazhang, "multistage detection in asynchronous code-division multiple access communications,” IEEE Transactions on Commun., vol. 38, pp. 509-519, April 1990.

16. P. Patel, J. Holtzman, "Analysis of a simple successive interference cancellation scheme in a DS/CDMA,” IEEE J. Select. Areas Commun., vol. 12, pp. 796-807, June 1994. 
17. A. Bahai and B. Saltzberg, Multi-Carrier Digital Communications, Theory and Applications of OFDM. New York: Kluwer, 1999.

18. J. Namgoong, T. Wong, J. Lehnert. "Subspace multiuser detection for multicarrier DS-CDMA," IEEE Transaction on Commun., pp. 1897-1908, Nov. 2000.

19. P. Tan, N.C. Beaulieu, "A comparison of DCT-based OFDM and DFT-based OFDM in frequency offset and fading channels," IEEE Trans. Commun., vol.54, no.11, pp.2113-2125, Nov. 2006.

20. Proakis, J.G.: 'Digital Communications' (McGraw-Hill Book Co, Singapore, 2001, $4^{\text {th }}$ edition.

21. X. Wang and H.V. Poor, "Blind adaptive multiuser detection in multipath CDMA channels based on subspace tracking," IEEE Trans. Signal Process., vol. 46, no. 11, pp.3030-3044, 1998

22. H. Liu and K. Li, “A decorrelating RAKE receiver for CDMA communications over frequencyselective fading channelsm," IEEE Trans. Commun., vol.47, no.7, pp.1036-1045, 1999

23. T. Liu, "Linearly constrained minimum variance filters for blind multiuser detection," IEEE Trans. Commun., vol. 51, no.10, pp.1649-1652, 2003

24. M. Tsatsanis and Z. Xu, "Performance analysis of minimum variance CDMA receivers," IEEE Trans. Signal Processing, vol. 46, pp.3014-3022, Nov. 1998.

25. Z. Xu, P. Liu and X. Wang, "Blind multiuser detection: from MOE to subspace methods," IEEE Trans. on Signal Processing, vol. 52, no. 2, pp. 510-524, February 2004.

26. H. Zhou, W.L. Woo and B.S. Sharif, "Expanded Subspace Approaches for Multiuser Detection for CDMA Multipath Frequency Selective Fading Channels," IEEE WCNM'05, Wuhan, China, vol.2, pp.635-638, Sept, 2005.

27. B. Burl: Linear optimal control: H2 and H[infinity] methods, Addison Wesley Longman, Inc., 1999.

28. M. S. Arulampalam, S. Maskell, N. Gordon, and T. Clapp, “A tutorial on particle filters for online non-linear/nongaussian bayesian tracking,” IEEE Trans. Signal Processing, vol. 50, no. 2, pp.174-188, 2002.

29. T.J. Lim, L.K. Rasmussen and H. Sugimoto: “An asynchronous multiuser CDMA detector based on the Kalman filter,” IEEE J. Select. Areas Commun., vol. 16, pp. 1711-1722, 1998. 


\section{FIGURES}
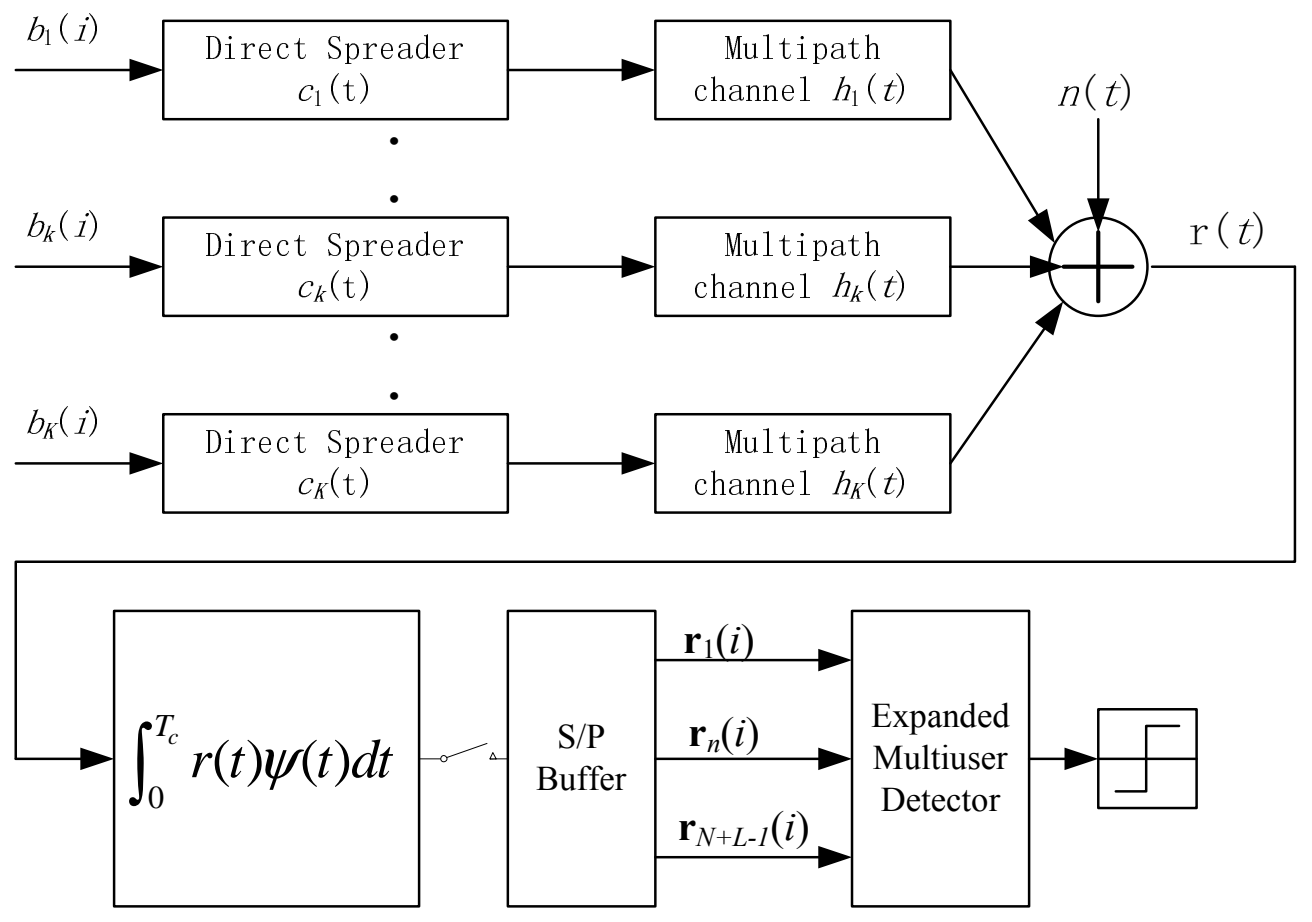

Figure 1: The system block diagram.

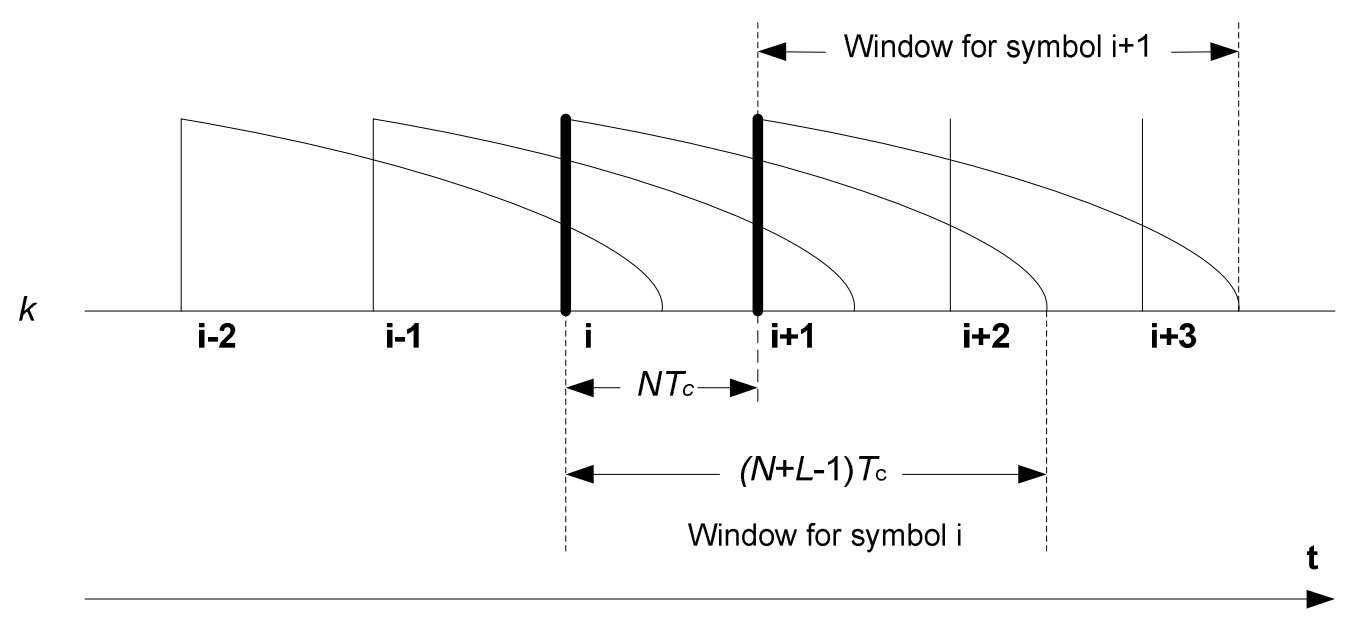

Figure 2: Diagram of the observation window for the synchronous multipath channel. 


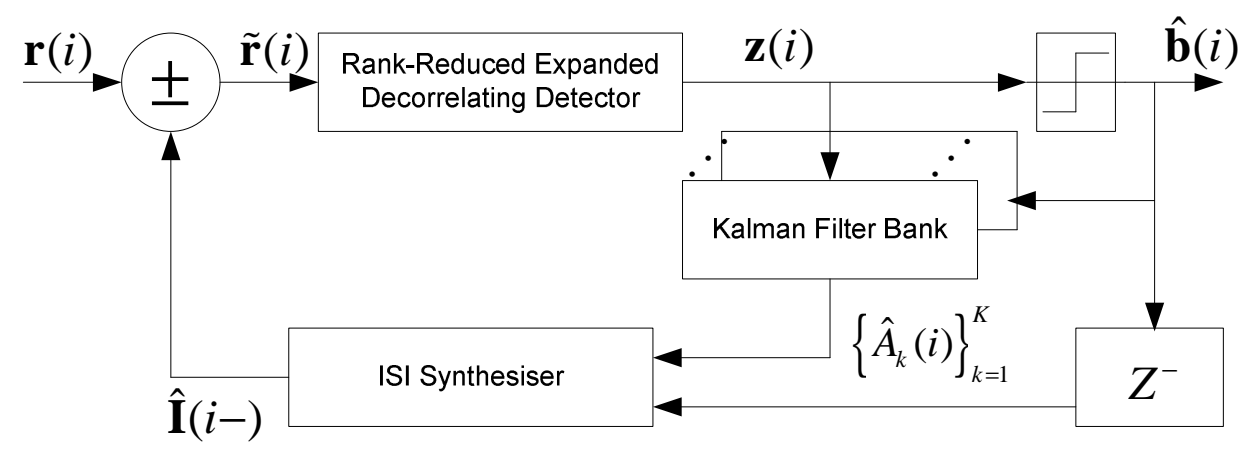

Figure 3: The hybrid detector including feed-forward interference cancellation, rank-reduced decorrelating detector and Kalman filter-based amplitude estimation (ExpLDD_IC1).

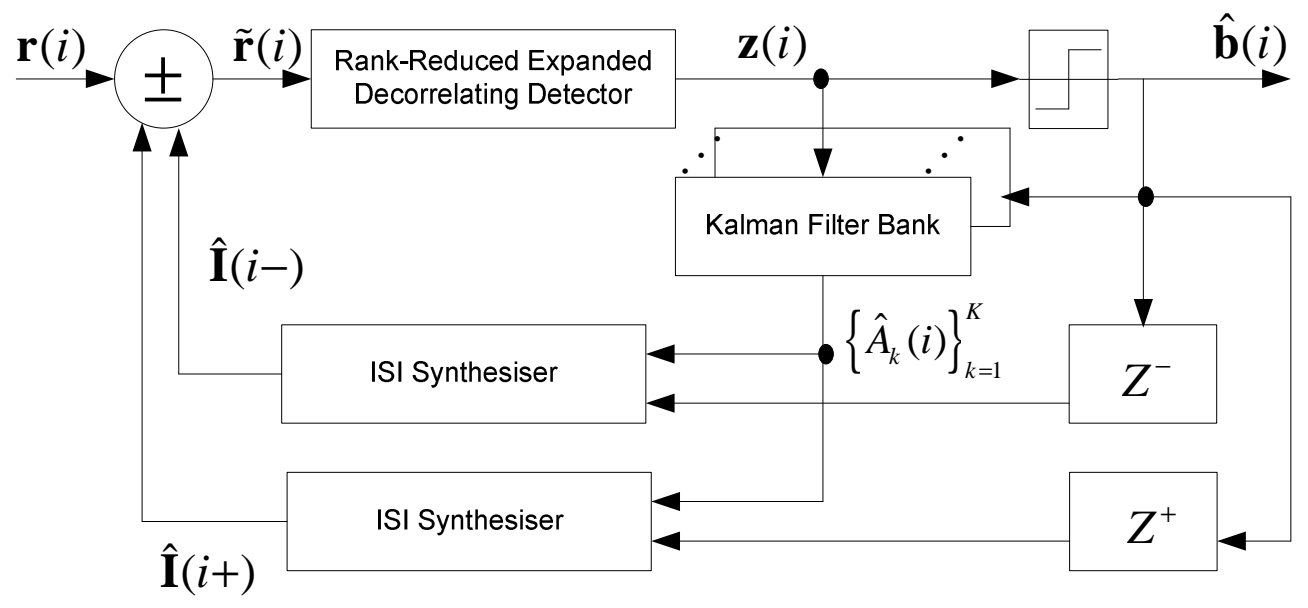

Figure 4: The iterative detection structure including feed-forward and feed-back interference cancellation, rank-reduced decorrelating detector and Kalman filter-based amplitude estimation (ExpLDD_IC2). 


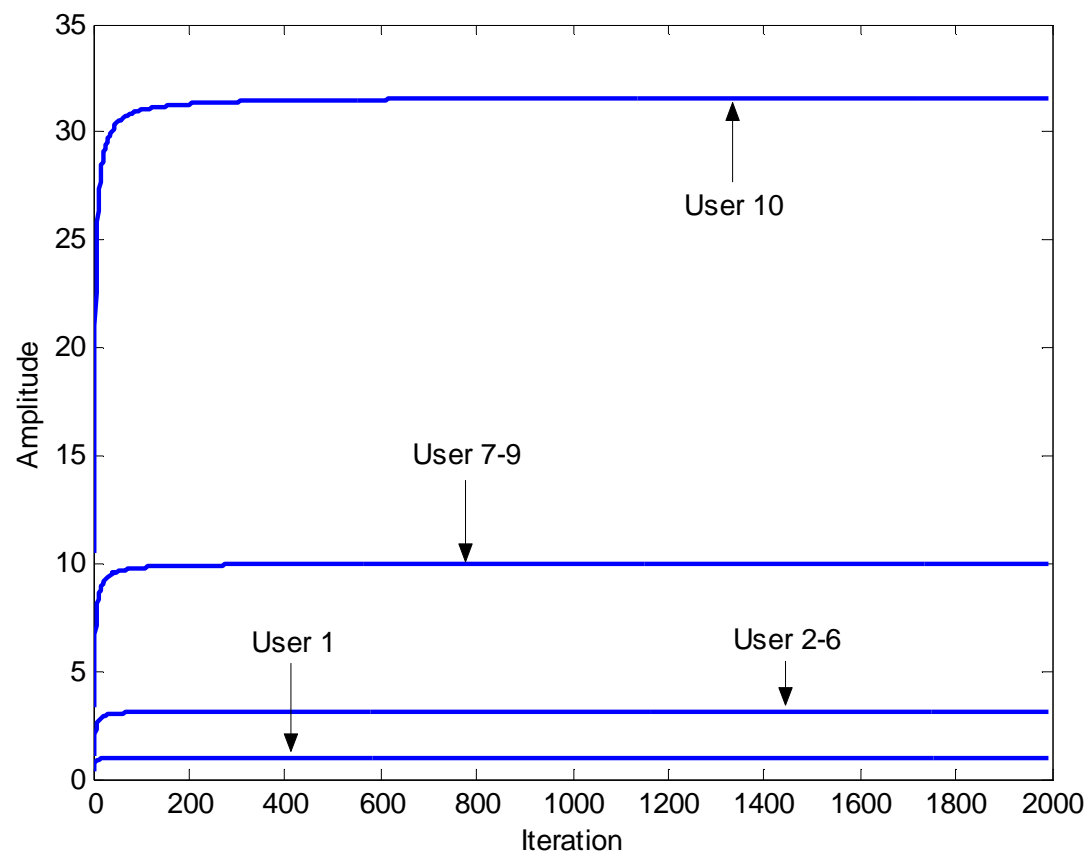

Figure 5: $(K=10, L=25, \mathrm{SNR}=20 \mathrm{~dB})$ Amplitude tracking for all users.

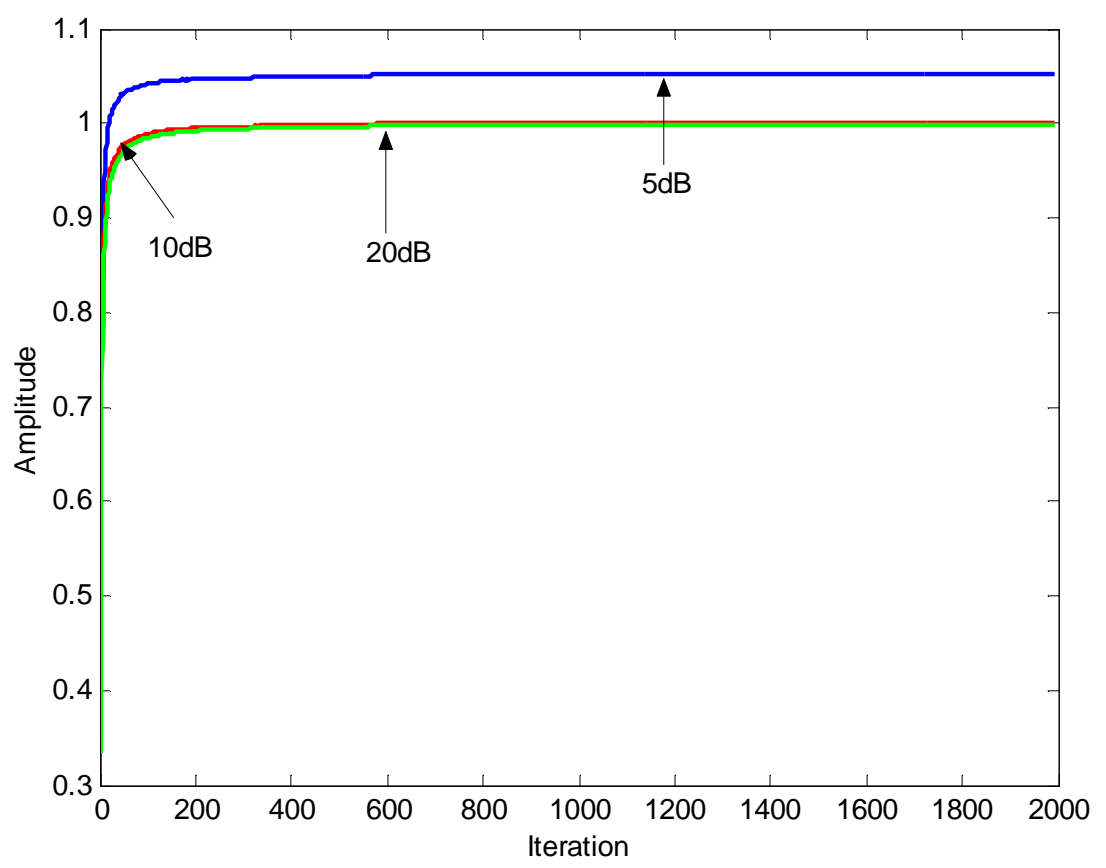

Figure 6: $(K=10, L=25)$ Amplitude tracking for user 1 at different SNR. 


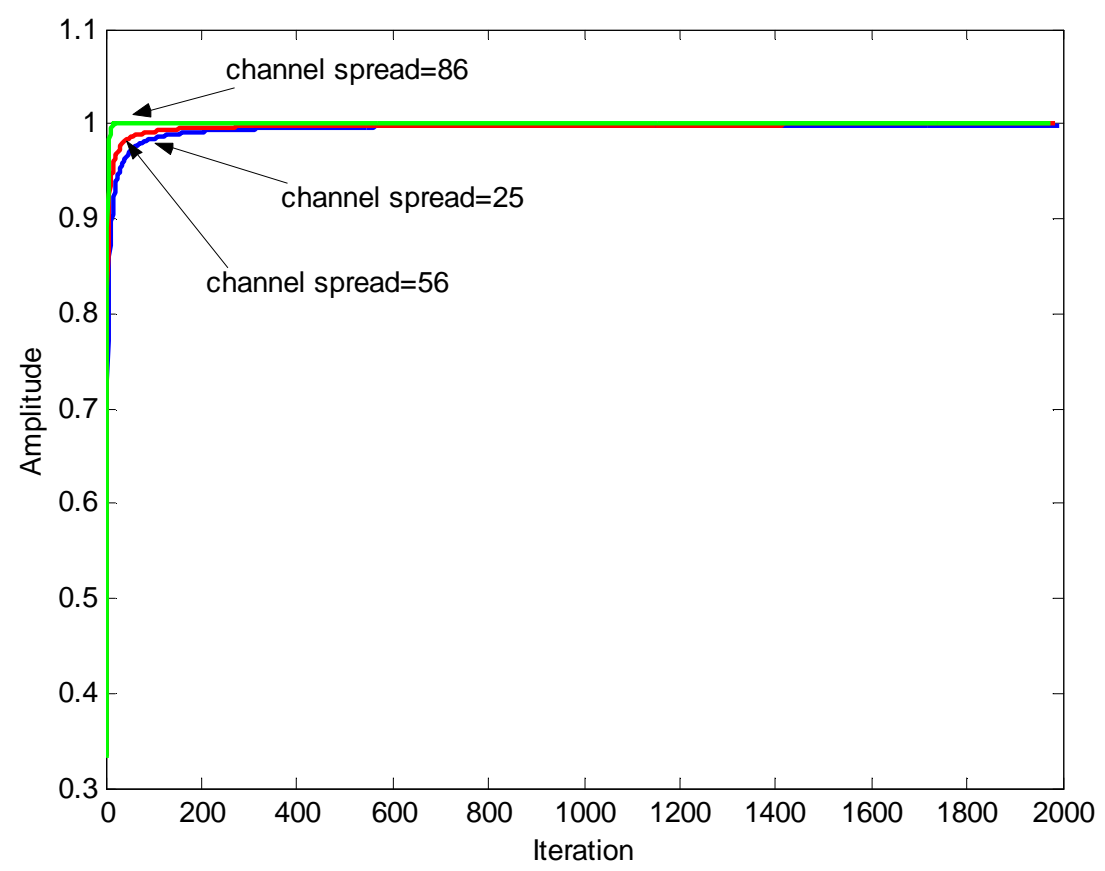

Figure 7: $(K=10, \mathrm{SNR}=20 \mathrm{~dB})$ Amplitude tracking at different channel spreads.

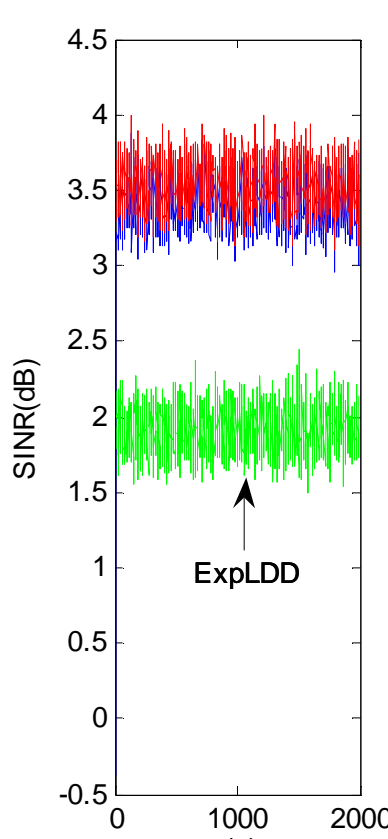

(a)
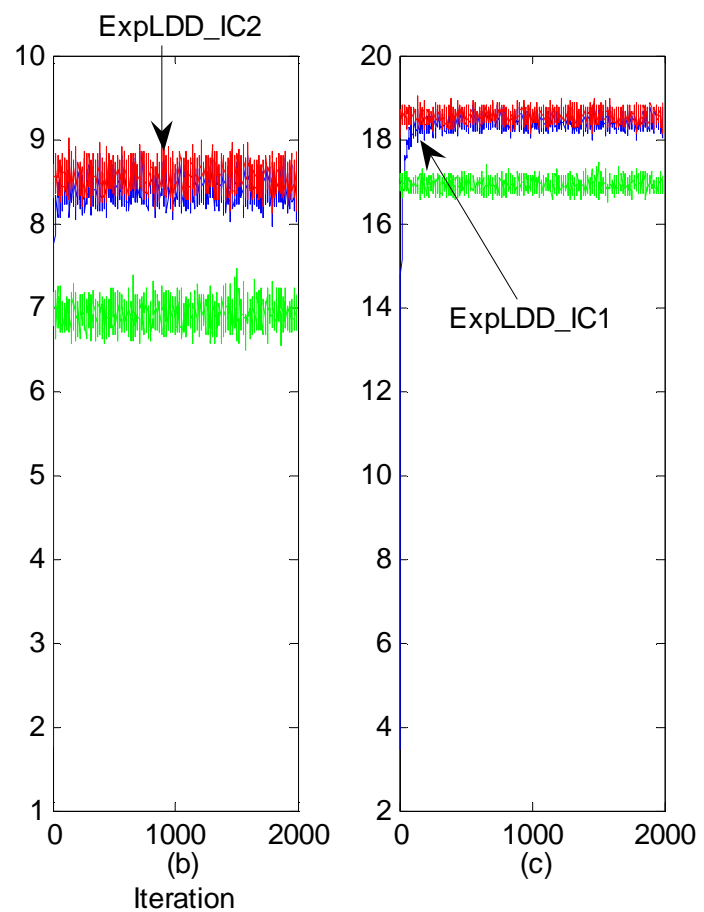

Figure 8: $(K=10, L=25)$ SINR convergence. (a) SNR=5dB; (b) SNR=10dB; (c) SNR=20dB. 


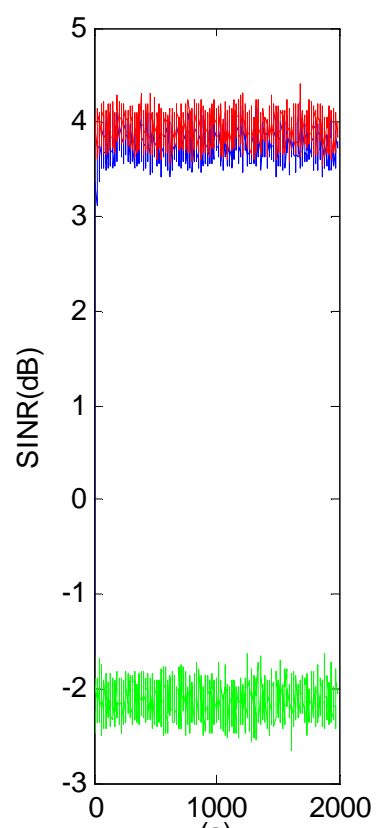

(a)
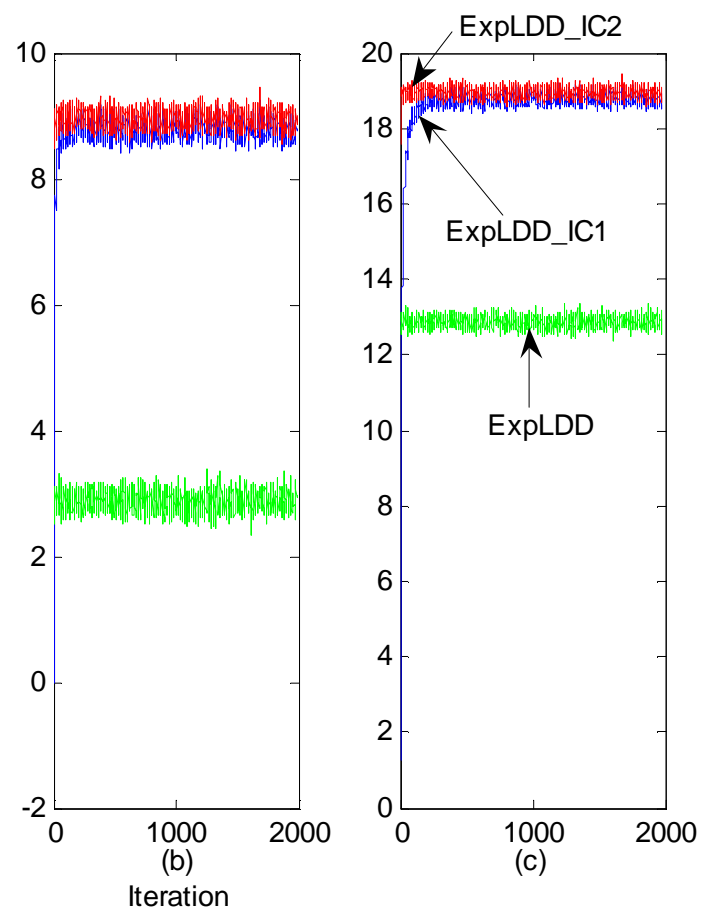

Figure 9: $(K=10, L=56)$ SINR convergence. (a) SNR=5dB; (b) SNR=10dB; (c) SNR=20dB.
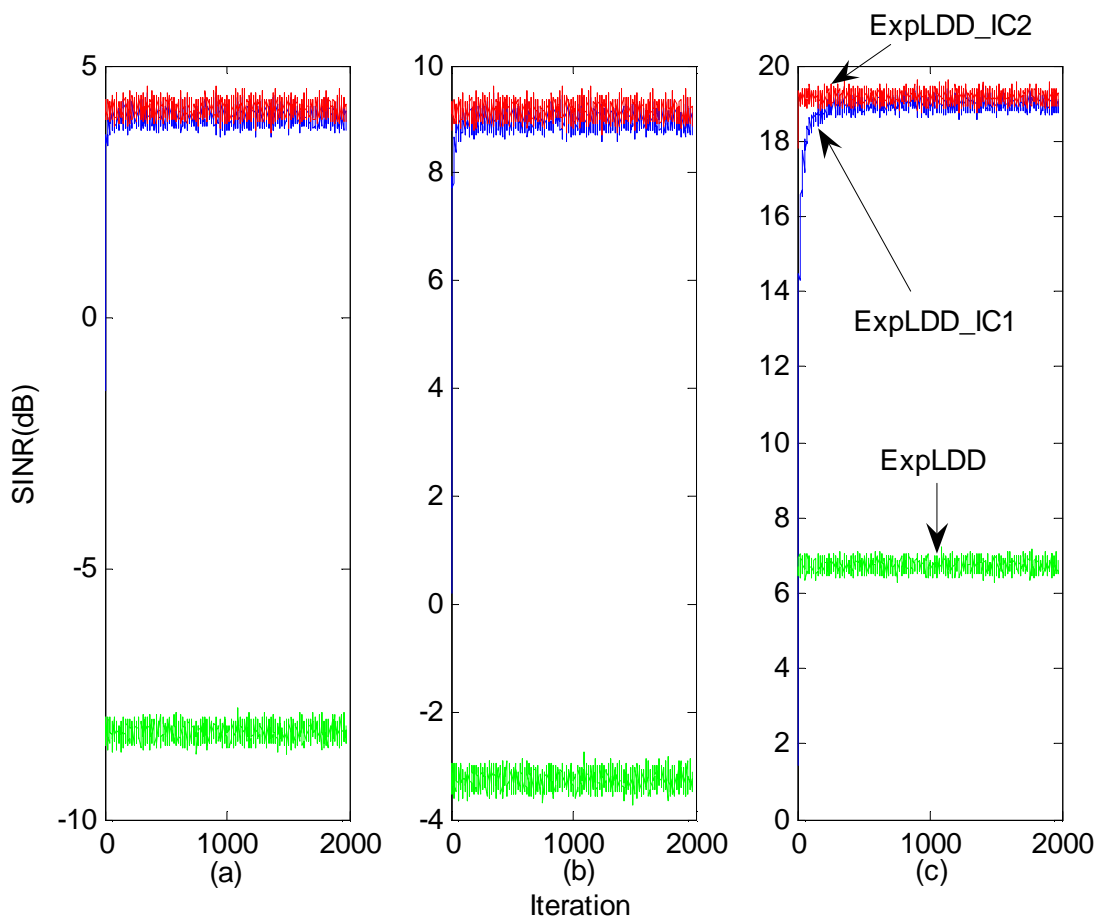

Figure 10: $(K=10, L=86)$ SINR convergence. (a) SNR=5dB; (b) SNR=10dB; (c) SNR=20dB. 


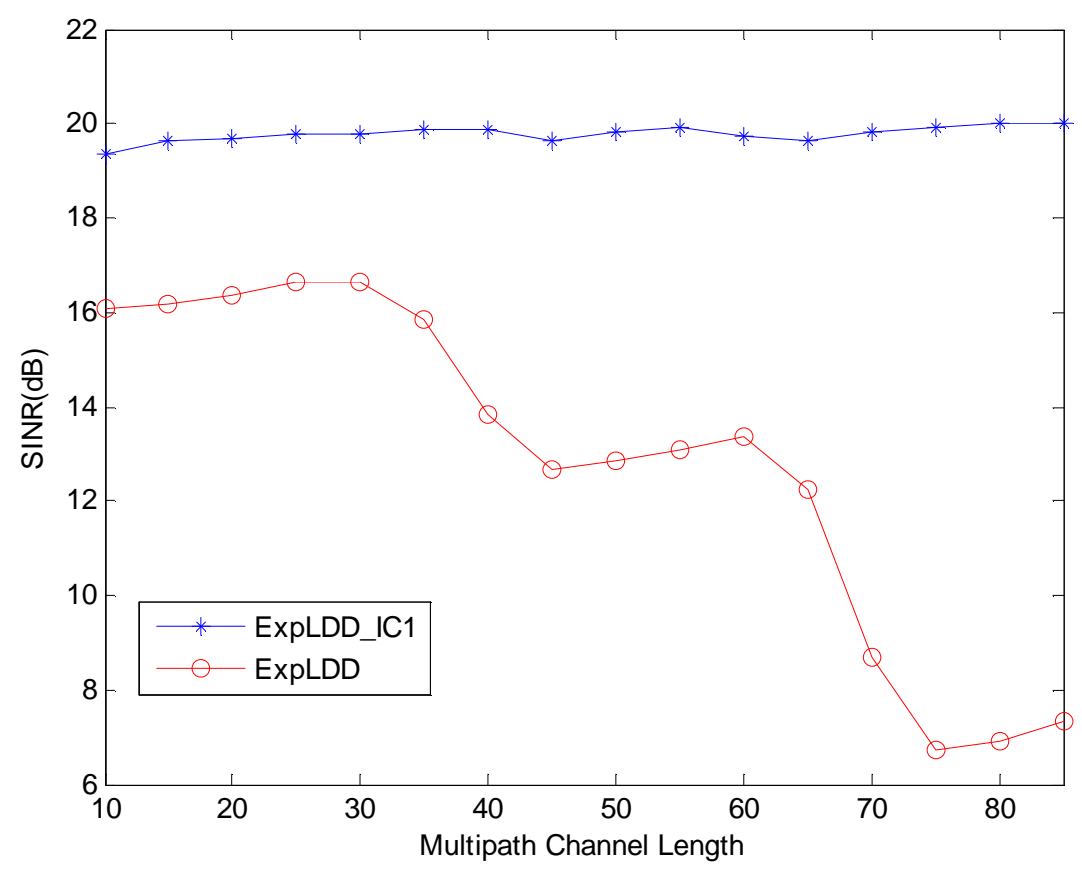

Figure 11: $(K=10, \mathrm{SNR}=20 \mathrm{~dB})$ SINR versus multipath channel spread.

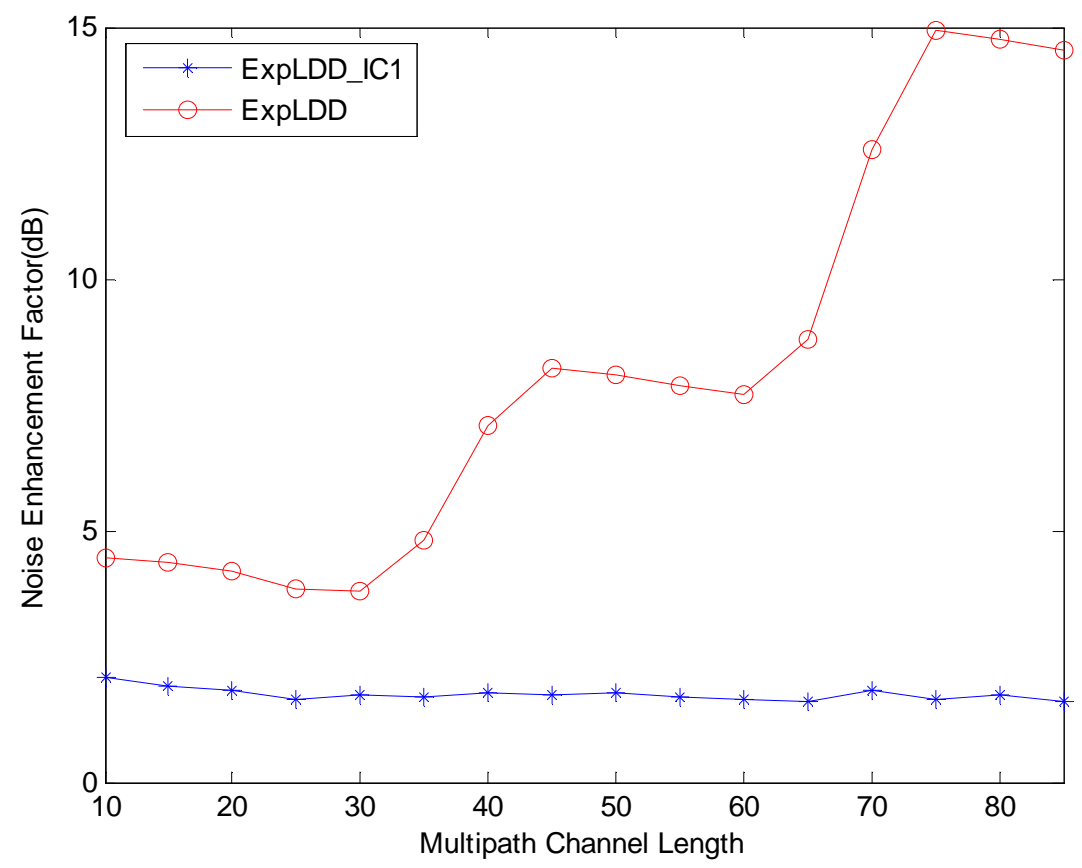

Figure $12(K=10)$ : Noise enhancement factor versus the multipath channel spread. 


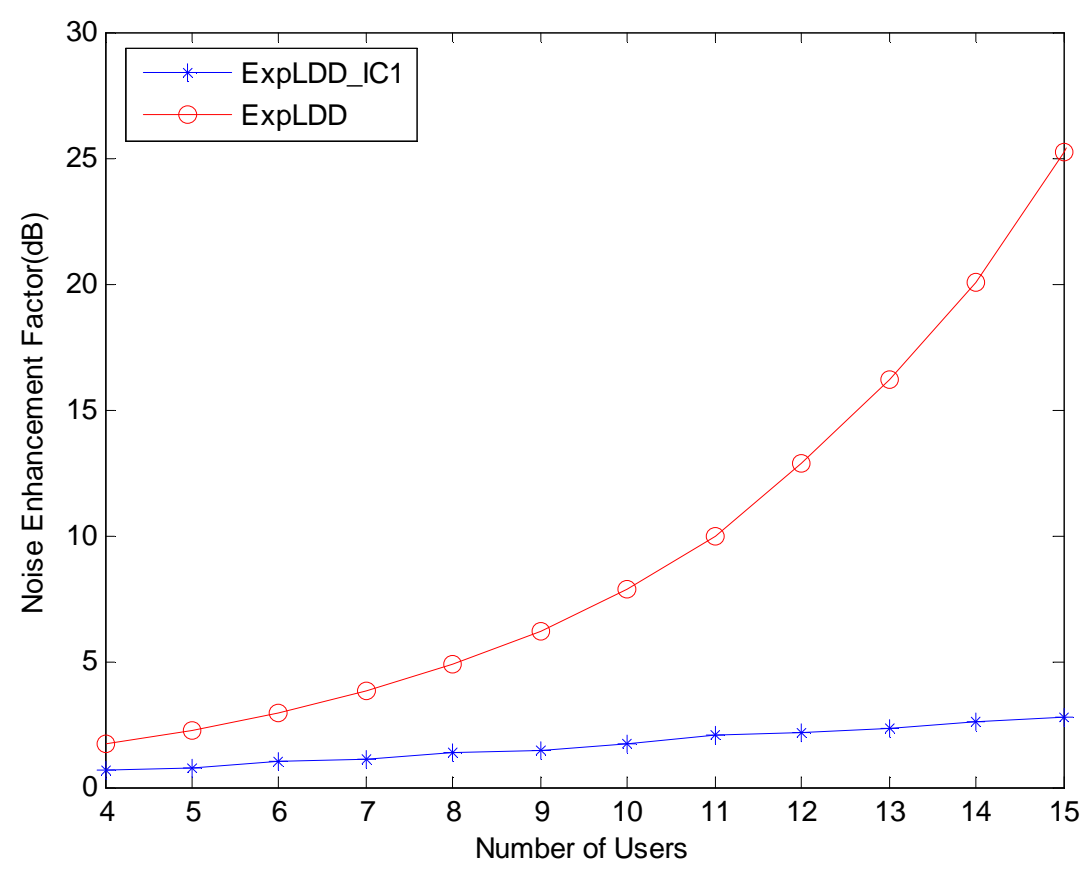

Figure 13 ( $L=56)$ : Noise enhancement factor versus the number of users.

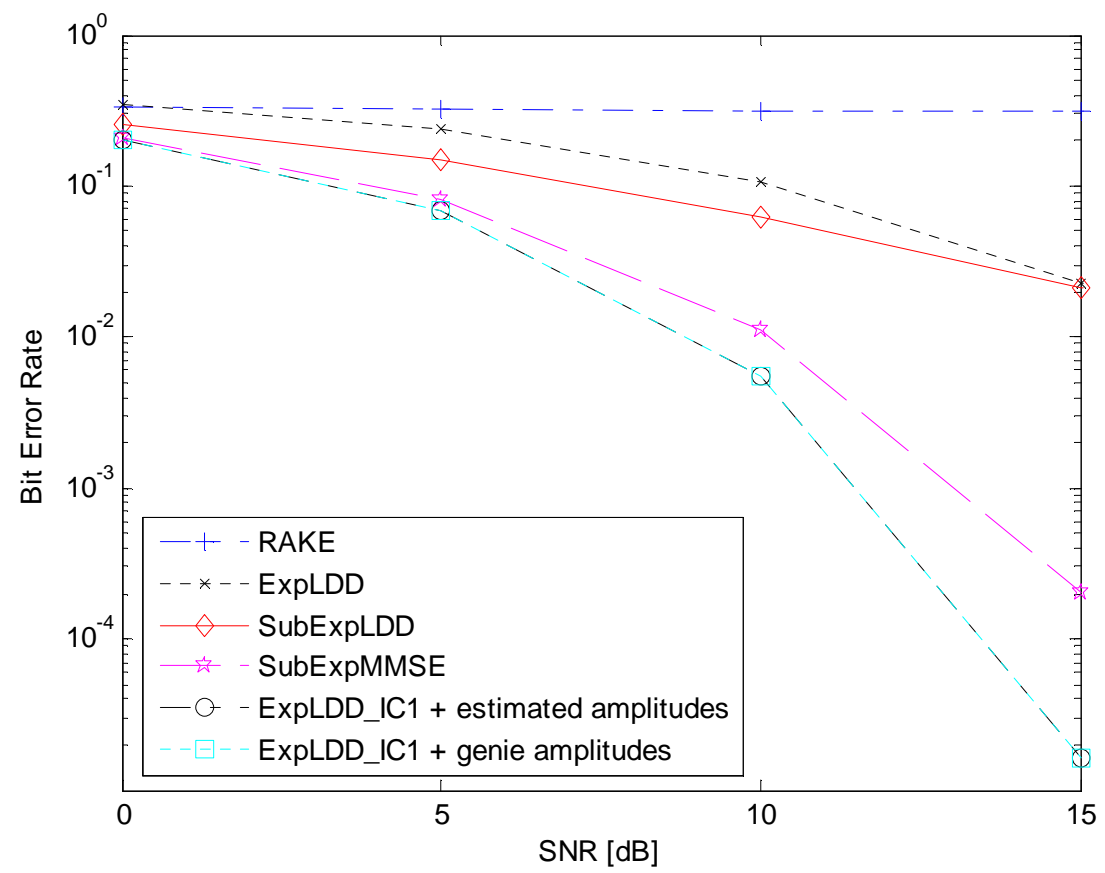

Figure 14: $(K=10, L=56)$ BER comparison of different multiuser detectors. 


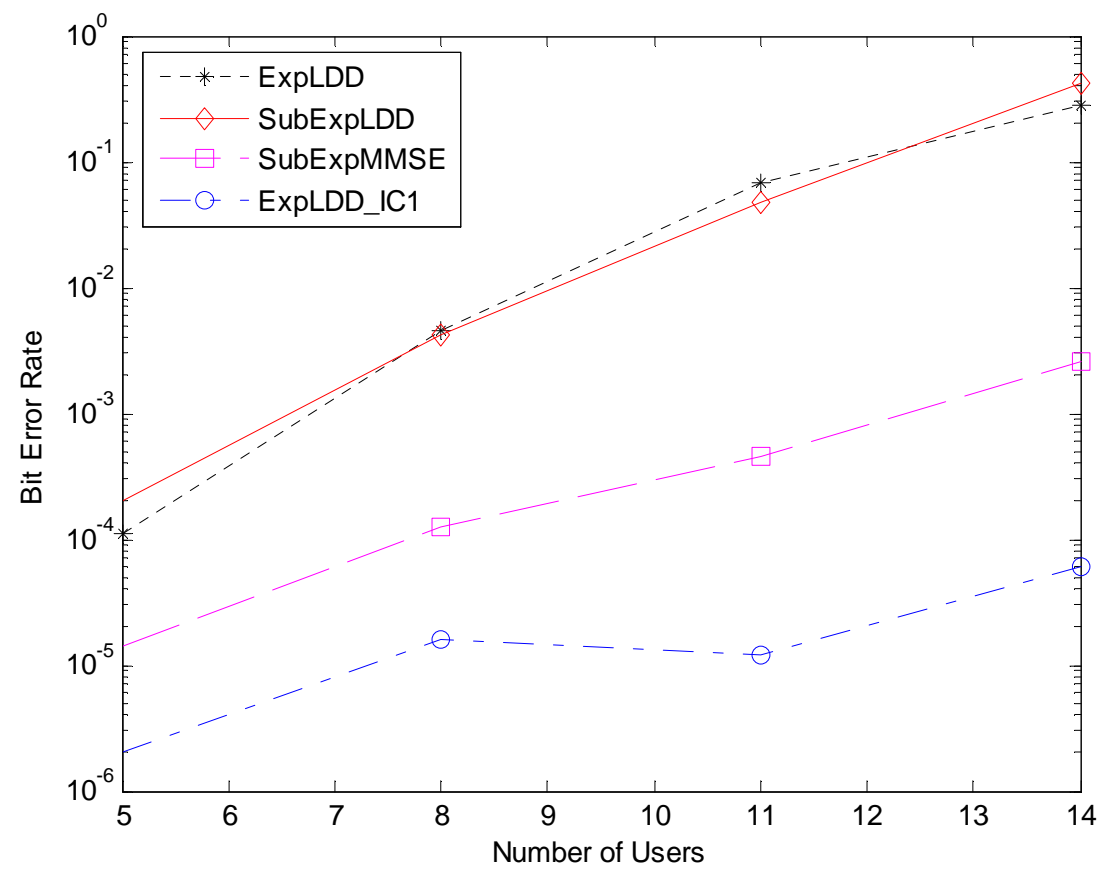

Figure 15: $(L=56, \mathrm{SNR}=15 \mathrm{~dB})$ Capacity comparison of different multiuser detectors. 